\title{
DOSAGE DE LA MÉTHIONINE DANS LES PRODUITS VÉGÉTAUX EMPLOYÉS DANS L'AIJIMENTATION ANIMALE
}

PAR

\section{Y. THUILLIER, G. FAUCONNEAU, S. de PRAILAUNE L. CHEVILLARD et J. ROCHE}

Laboratoire de Biochimie générale et comparée (Collège de France) Laboratoire de Biochimie des Vitamines (Ecole des Hautes Etudes) et Institut National de la Recherche Agronomique

Le dosage de méthionine par des méthodes chimiques, dont la spécificité est médiocre, s'avère difficile dans les produits végétaux ; aussi avons nous cherché à le réaliser par voie microbiologique.

Essais de microorganismes. - L'emploi de trois microorganismes: Lactobacillus arabinosus I $7 / 5$, Leuconostoc mesenteroides $\mathrm{P}$. 60 et Lactobacillus fermenti 36 , a été étudié en titrant l'acidité totale libérée par chacun de ceux-ci dans des conditions standards : la méthionine est fournie par les échantillons ou sous forme $L\left({ }^{1}\right)$. Comme la plupart des produits végétaux sont pauvres en méthionine, nous avons évité les pertes pendant la stérilisation en autoclavant séparément les milieux non glucosés, les solutions de glucose, et en ajoutant ensuite aseptiquement ces dernières Riesen (3I), Camien (9), Chevillard (I2).

\section{LACTOBACILLUS ARABINOSUS $17 / 5$ A. T. C. C. 8014}

Cet organisme a été préconisé par de nombreux auteurs pour le dosage de la méthionine. De plus Dunn (I5), Shankmann (33), Mahadevan (28), MONDOLFO (29), SAUBERIICH (32), ont obtenu des résultats cohérents pour le dosage de la méthionine dans l'urine et dans les protéines purifiées en utilisant divers milieux: Shankmann modifié par Dunn (I5),

(1) Nous remercions les professeurs W. C. ROSE (Illinois) et WRETLIND (Stockholm) de nous avoir obligeamment adressé les échantillons de L-méthionine nous ayant servi de corps de référence. 
Sauberlich (32), Mahadevan (28) et Henderson (2I). La spécificité de ce microorganisme a été étudiée par CAMIEN (8) quant à l'utilisation des isomères optiques, à l'aide du milieu de SHANkMANN enrichi en vitamines. En présence de quantités suffisantes de pyridoxal ( $\mathrm{y}$ ug p. cent), de pyridoxamine (Io y.g $\mathrm{p}$. cent), la $\mathrm{D}$-méthionine a une activité à peu près équivalente à celle de la L-méthionine. Alors qu'en 1'absence de ces formes de vitamine $B_{6}$, nil'isomère $\mathrm{D}$ ni la formyl-D-méthionine ne possèdent d'activité (SpIEs 37) ; par ailleurs la DL-éthionine est douée d'un pouvoir inhibiteur égal vis-à-vis de l'utilisation de la $\mathrm{D}$ et de la $\mathrm{L}$-méthionine. L'un des dérivés d'oxydation, la DL méthioninesulfoxyde présente une activité comparable à celle de la L-méthionine, tandis que l'autre, la DL-méthioninesulfone (ou la caséine oxydée) en est dépourvue et, de plus, empêche 1'utilisation de la DL-méthionine-sulfoxyde (CAMIEN II). La D-méthionine et la DL-méthionine-sulfoxyde seraient transformées en $\mathrm{L}$-méthionine avant d'être utilisées et les formes de vitamine $B_{i}$ mentionnées plus haut seraient les coenzymes permettant cette réaction enzymatique, qui pourrait par ailleurs être inhibée par 1a DL-méthioninesulfone. DUnn et ses collaborateurs ( I 7 ) concluent de leur étude théorique sur les besoins des bactéries lactiques, au caractère indispensable de la I,-méthionine pour Lactobacillus arabinosus dans des conditions particulières: "Le milieu et les échantillons ne doivent contenir un excès ni de cystine, ni de pyridoxal (ou pyridoxamine), qui contribueraient à la croissance en l'absence de méthionine" (CAMIEN II). Ces résultats sont en accord avec ceux d'Evans (I8). Lactobacillus arabinosus présente une croissance appréciable sans méthionine (blanc: 3,7), mais exige cette dernière pour une croissance optimum. De plus, Evans utilisant le milieu de ScHWEIGERT sans choline, trouve que l'homocystine avec ou sans choline ne peut remplacer la méthionine qu'à des concentrations supérieures à celles de I-méthionine nécessaires.

Choix du milieu. - I e milieu et les modalités de culture (DUNN I7) (temps d'incubation, inoculum... etc.) peuvent faire varier les besoins en un aminoacide; aussi ces facteurs sont-ils importants dans l'emploi d'un microorganisme pour le dosage d'un acide aminé. I_e milieu de HorN (22) n'a pu être retenu, car malgré une sensibilité satisfaisante (I à ro yg pour un volume final de Io cc) la consommation de $\mathrm{NaOH}$ o, I N n'a été que de 6 cc pour la croissance maximum ( 8 j.g et au-dessus). CAmin (II) utilisant le milieu de SHankman (33) enrichi en vitamines a montré l'identité d'activité de la L-méthionine et de la DL-méthioninesulfoxyde, ainsi que le pouvoir inhibiteur de la DL-méthioninesulfone. L'emploi de ce milieu pour le dosage de la méthionine dans divers échantillons s'est avéré délicat. I a méthode est très sensible (o,2 à $2 \mu \mathrm{g})$, mais la quantité d'inoculum à introduire dans le milieu demande à être trop exacte- 
ment mesurée pour que cela soit facilement réalisable. En effet, cette quantité optimum d'inoculum (mesurée en densité optique par opacimétrie sous une épaisseur de $3 \mathrm{~cm}$ ) est variable, même si 1'on contrôle exactement le temps d'incubation de l'inoculum et sa composition. Des quantités trop faibles ne permettent pas une croissance suffisante, alors que des quantités trop fortes augmentent proportionnellement la valeur des blancs et rendent tout dosage impossible. CAMIEN et DUNN signalaient seulement la haute valeur des blancs (II) (tableau I). Ce milieu,

\section{TABLEAU I}

Influence de la dilution de l'Inoculum sur la croissance du L. arabinosus exprimée en cc de $\mathrm{NaOH}$ o,or $N$ par cc de milieu final (2 et $3^{\text {es }}$ lignes horizontales).

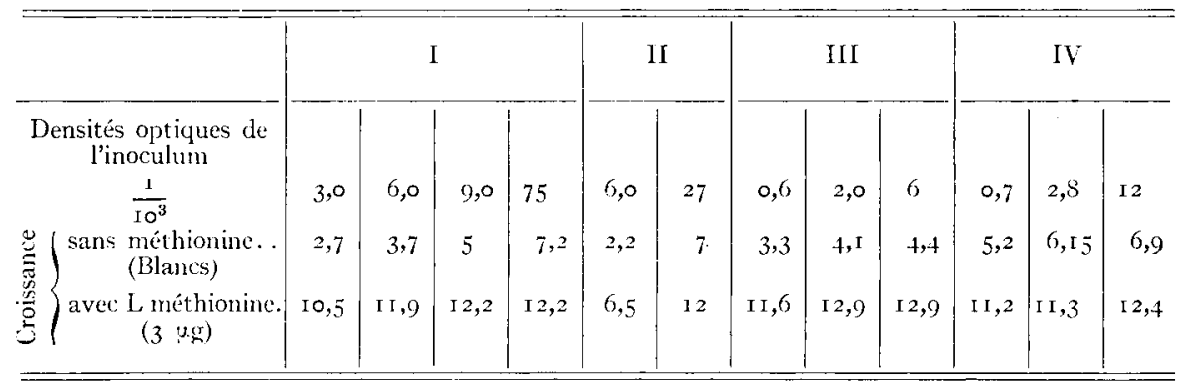

riche en vitamines du groupe $B$ et en choline, est de composition optima en aminoacides. Il permet généralement la croissance du Lactobacillus arabinosus en l'absence de méthionine (en présence de faibles quantités de cystéine), à moins que des conditions particulières d'ensemencement ne limitent artificiellement sa croissance. Ainsi, ce milieu ne peut fournir une méthode de dosage fidèle.

Nous avons utilisé comparativement le milieu préconisé par MAHADEVAN dépourvu de choline et de composition banale en aminoacides; l'apport d'acides aminés de l'échantillon à doser ne provoque pas de perturbations (MoNDOLFo (29). Mais ce milieu, comparativement à celui de HORN (22) est très pauvre en vitamines du groupe $B$ et nous n'avons pu l'utiliser tel quel pour le dosage de la méthionine dans les levures de distillerie (tableau II et courbe I). L'augmentation de certaines vitamines du groupe B : B I (xIO) PP (x4) Biotine (x5) Acide folique (x2,5) nous a permis d'obtenir des résultats cohérents : les blancs restent bas ( $\mathrm{I}, 5$ à 2,5 cc de $\mathrm{NaOH}$ o,or $\mathrm{N}$ par cc de milieu-final) et les courbes standard et échantillons coïncident dans la limite des erreurs expérimentales admises \pm 3 p. cent) tableau III, et courbe II. 


\section{TABLEAU II (Courbes I).}

Courbes standard et échantillon de levure (Milieu Mahadevan non enrichi en vitamines) volume final $3 \mathrm{cc}$. Organisme utilisé: Lactobacillus arabinosus $\mathrm{I} 7 / 5$.

\begin{tabular}{|c|c|c|c|}
\hline $\begin{array}{l}\text { Echantillon dilué } \\
\text { cc }\end{array}$ & $\begin{array}{l}\mathrm{NaOHI} 0,0_{3} \mathrm{~N} \\
\text { en cc }\end{array}$ & $\begin{array}{l}\text { L. Méthionine } c c \\
(24 \mu g \text { par } c c)\end{array}$ & $\underset{\text { en cc }}{\mathrm{NaOH}}$ \\
\hline $\begin{array}{l}0,0 \\
0,2 \\
0,4 \\
0,6 \\
0,8 \\
\mathrm{I}\end{array}$ & $\begin{array}{c}2,3 \\
4,85 \\
6,9 \\
8,3 \\
9,3 \\
\text { I0,I }\end{array}$ & $\begin{array}{l}0 \\
0,1 \\
0,1 \\
0,2 \\
0,3 \\
0,4 \\
0,5 \\
0,6 \\
0,7 \\
0,8 \\
0,9 \\
\text { I }\end{array}$ & $\begin{array}{c}2,3 \\
4,8 \\
6,4 \\
7,5 \\
8,1 \\
8,5 \\
9,0 \\
9,35 \\
9,60 \\
10,0 \\
10,15\end{array}$ \\
\hline
\end{tabular}

Tableau III (Courbe II).

Courbe standard obtenue avec L. arabinosus. (Volume final $3 \mathrm{cc}$, 44 heures d'incubation.

\begin{tabular}{c|c}
\hline $\begin{array}{c}\text { Courbe standard } \\
\text { de }\end{array}$ & $\begin{array}{c}\text { NaOH o,o3 N } \\
\text { employés } \\
\text { Mour le dosage } \\
\text { en cc }\end{array}$ \\
\hline & \\
\hline 0 & $\mathrm{I}, 85$ \\
$\mathrm{I}, 6$ & 3,90 \\
3,2 & 5,20 \\
4,8 & 6,40 \\
6,4 & 7,20 \\
8,0 & 7,57 \\
9,6 & 8,00 \\
$1 \mathrm{I}, 2$ & 8,45 \\
$\mathrm{I} 2,8$ & 8,90 \\
14,4 & 9,25 \\
$\mathrm{I} 6$ & 9,55 \\
\hline \hline
\end{tabular}

Mode opératoire. - I $\mathrm{I}_{\mathfrak{a}}$ méthode améliorée par la stérilisation séparée du glucose n'a pu être utilisée avec la technique suivie pour la cystine (Chevillard I2), car le milieu concentré (I/3) ne peut être préparé, les acides aminés dont ce milieu est relativement riche formant un abondant précipité au moment de la neutralisation. On opère sur $3 \mathrm{cc}$ et l'inoculum est préparé avec I5 : $\mathrm{g}$ de $\mathrm{L}$-méthionine pour un volume total de $9 \mathrm{cc}$. L'inoculum est incubé 20 heures, centrifugé, remis en suspension dans de l'eau physiologique stérile, puis dilué io fois avant l'ensemencement qui se fait avec une goutte par tube, à la pipette Pasteur. L'incubation des tubes doit être au minimum de 44 heures (DunN I7). Le chlo- 

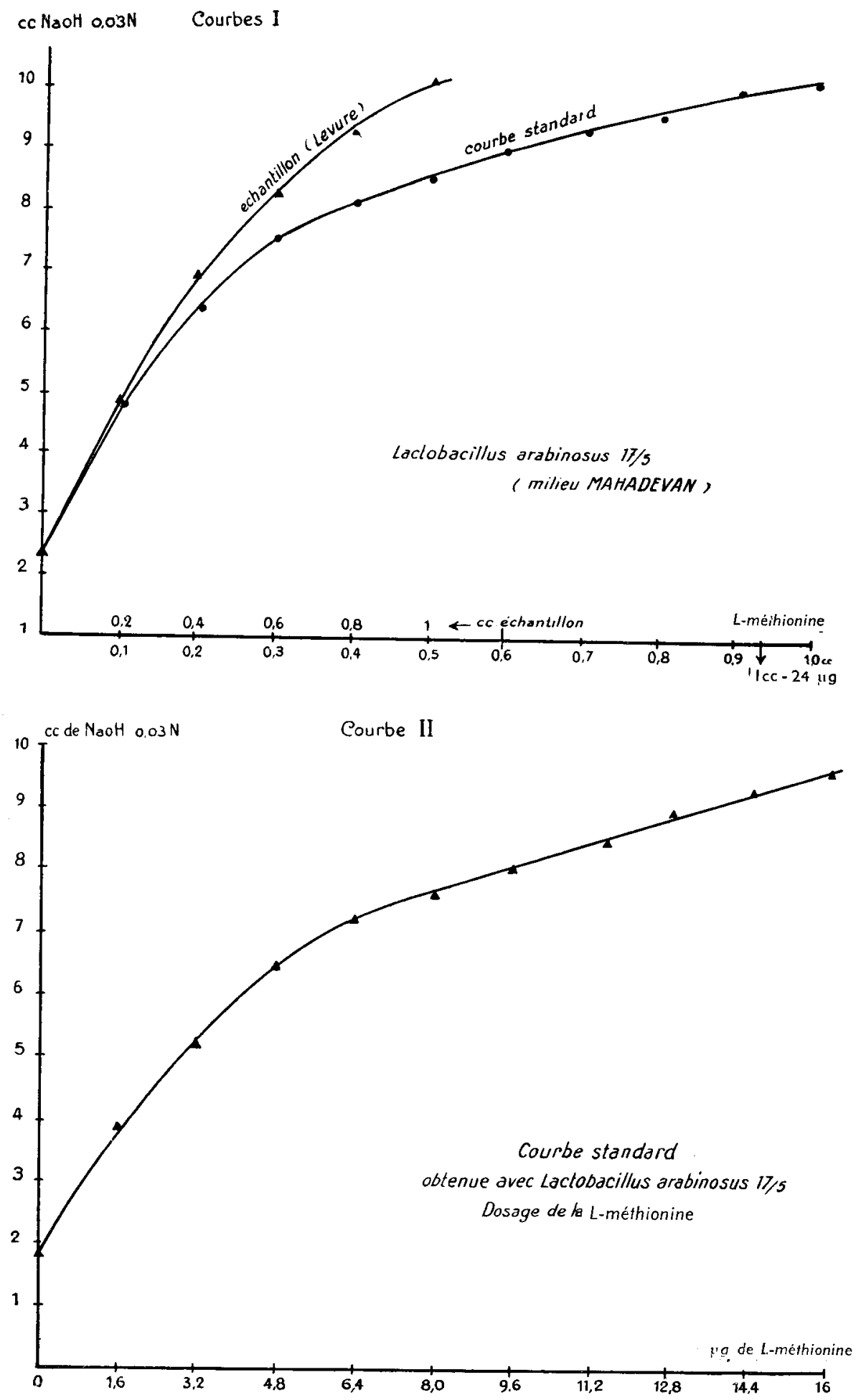
rure de sodium provenant de la neutralisation des hydrolysats par la soude ne gêne pas la croissance du microorganisme, si la concentration est inférieure à $20 \mu \mathrm{g}$ pour $3 \mathrm{cc}$ de milieu final.

Précision de la méthode. - Tous les échantillons analysés ont satisfait aux critères d'application des méthodes microbiologiques (SNELL 35, 36), étant donné, les résultats cohérents pour des concentrations croissantes d'échantillon. L'analyse statistique de ces résultats : 5 concentrations croissantes (en double), nous permet de définir la variabilité des résultats pour la luzerne $\mathrm{N} \%: 2,5$.

Concentrations croissantes (en cr) $\ldots \ldots \ldots \ldots \ldots \ldots \ldots \ldots$
En $\%$ du maximum de la courbe standard par $c \ldots \ldots \ldots$
$\sigma=3,16$

Le calcul de la variabilité a été fait sur la totalité des valeurs indépendamment de la concentration.

Calcul en tenant compte des dilutions de 1'échantillon.

$\mathrm{Si}$

$$
60,2 \times 0,016 \times 2=I, 93 \mu \mathrm{gg} / \mathrm{gramme} .
$$

$$
\begin{gathered}
\mathrm{P}=0,05 \\
2 \times \mathrm{I}, 05 \times 0,0 \mathrm{I} 6 \times 2=0,07 \\
\mathrm{I}, 93 \pm 0,07
\end{gathered}
$$

La précision est donc de $\pm 3,5 \%$, elle a été vérifiée pour cet échantillon au cours de 4 dosages effectués dans les mêmes conditions à plusieurs mois d'intervalle:

$$
\text { I,93 2,O I,85 2,O }
$$

\section{LEUCONOSTOC MESENTEROIDES P. 60 (A. T. C. C. 8042)}

L'étude théorique des besoins de ce microorganisme a été faite par divers auteurs (DUNn (I3), EVANS (I8), STFELF (38)), ell utilisant des milieux différents. La $\mathbf{L}$-méthionine paraît toujours être strictement indispensable quelle que soit la quantité de cystine présente dans le milieu. En effet, Evans (I8), d'une part, avec le milieu de Riesen (30) et LymANN (26), d'autre part, ont montré que l'homocystine ne peut remplacer la méthionine même en présence d'un excès de cystine et de choline. De plus, Dunn (I5) et Camien (Io) et Lymann (26) concluent de leurs études que, quelles que soient les vitamines apportées par le milieu, la D-méthionine n'est pas utilisée par le Leuconostoc. Cependant STEELE (38) trouve à celui-ci une activité égale au I/Io de celle de l'isomère L. Des essais comparés de la L-méthionine (Wretlind) et de la DL-méthionine (Interchemical Corporation) nous ont conduit à attribuer à la forme 
L essayée 85 p. cent $\pm 3,5$ p. cent de l'activité de la L-méthionine théoriquement contenue dans la forme DL. Cette différence avec les résultats de STEELE pourrait être due à des traces de l'isomère $\mathrm{D}$ dans la forme I essayée.

Nous avons d'autre part, poursuivi cette étude de la spécificité du L. mésenteroides par 1'activité des dérivés d'oxydation (tableau IV). La DL-méthioninesulfoxyde a une activité assez faible sur la croissance du $L$. mésenteroides pour des concentrations inférieures à $I_{5} \mu \mathrm{g}$, mais cette activité augmente notablement au-dessus de cette concentration. La DL-méthioninesulfone ne possède aucune activité sur la croissance (activation, ou inhibition) du L. mésenteroides, même si le milieu en contient Io fois la teneur en méthionine (300 $\mu \mathrm{g}$ de DI--méthioninesulfone par tube d'un volume total de $3 \mathrm{cc}$ ). On peut conclure de cette étude que 1a L-méthionine est strictement indispensable au Leuconostoc mésentéroides et quelle ne peut être remplacée que partiellement par le DLméthioninesulfoxyde $(20 \mathrm{p}$. cent de l'activité pour les concentrations inférieures à I5 ug par tube).

\section{TABLEAU IV}

Influence des dérivés d'oxydation de la DL-méthionine sur la production d'acide lactique du Leuconostoc mésenteroides P. 60.

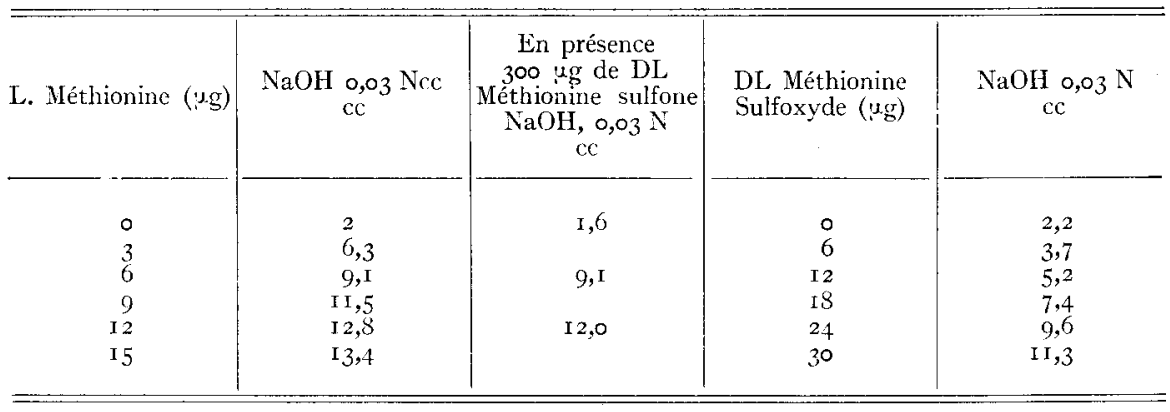

Beaucoup d'auteurs ont préconisé l'emploi de ce microorganisme pour le dosage de la L-méthionine (Dunn (I5), STEELE (38), BartonWright (4), Singleton (34), mais seul le dernier l'a appliqué à des végétaux frais avec le milieu de STOCKES modifié (39).

Mode opératoire. - La méthode utilisant L. mésenteroides avec le milieu D de Dunv est assez sensible (I à I $2,5 \mathrm{pg}$ ) en utilisant la stérilisation séparée du glucose et le mode opératoire déjà décrit pour ce microorganisme (Chevillard (I2), à la différence que l'on centrifuge l'inoculum avant de l'utiliser. Les blancs sont toujours bas : I,5 à 2,5 cc. Na OH, $0,03 \mathrm{~N}$ pour $3 \mathrm{cc}$. de milieu final courbe III et tableau $\mathrm{V}$. 


\section{Tableau V (Courbe III)}

Courbe standard obtenue avec Leuconostoc Mésenteroides P. 60 pour le dosage de la L-méthionine. Volume total $3 c c, 5$ jours d'incubation.

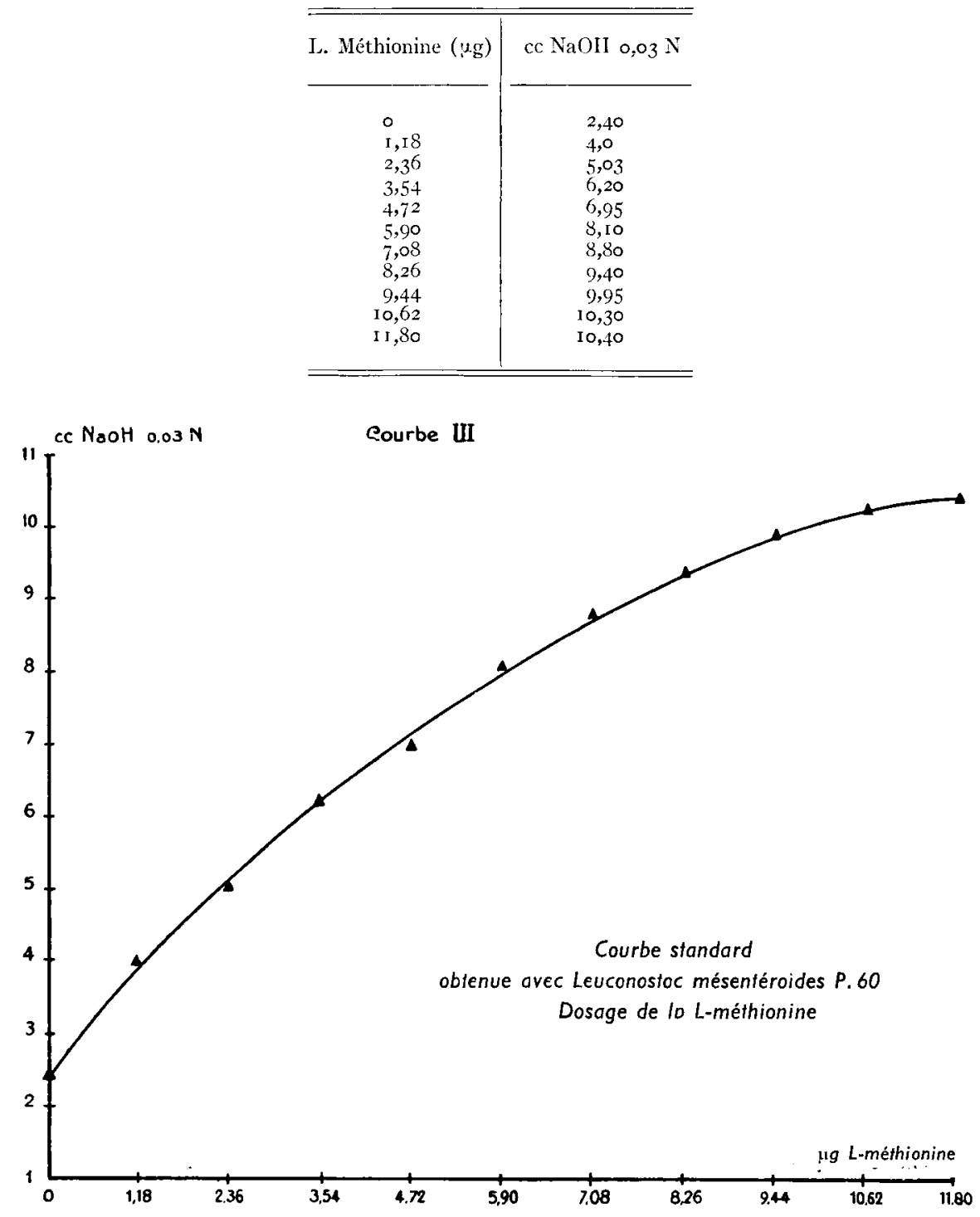

Limite et précision de la méthode. - I 'utilisation de cette méthode très spécifique n'a pu être généralisée à tous les échantillons analysés. Le dosage de la méthionine s'avère impossible dans certains fourrages pauvres en matières azotées $(\mathrm{N} \%<2,5 \%)$, par suite de la présence d'inhibiteurs dans les échantillons. Les deux courbes standard et échan- 
tillons ne sont pas superposables comme 1'indique le tableau ci-dessous, les chiffres sont exprimés en $\%$ du maximum de la courbe standard pour une même quantité d'échantillon dilué (I cc) et correspondant à des concentrations croissantes de ce dernier.

\begin{tabular}{|c|c|c|c|c|c|c|}
\hline & $\mathrm{N} \%$ & 0,2 & 0,4 & 0,6 & 0,8 & I \\
\hline Luzeme ...... & 2,2 & 1,0 & 0,98 & 0,90 & 0,84 & 0,75 \\
\hline ........ & 1,2 & 0,68 & 0,52 & 0,48 & 0,44 & 0,42 \\
\hline Luzeme + Méthionine & 2,2 & 0,94 & 0,92 & 0,86 & $0,8 \mathrm{I}$ & 0,76 \\
\hline
\end{tabular}

Cette inhibition persiste pour une dilution plus grande, même si la L-méthionine est ajoutée en surcharge. Elle ne peut être attribuée au chlorure de sodium provenant de la neutralisation des échantillons, la concentration en ce sel était la même dans d'autres échantillons où aucune inhibition n'apparaissait (luzerne $2,5 \%$ et $4,3 \%$ d'N).

L'analyse statistique des résultats satisfaisants aux critères d'application de la méthode microbiologique (superposition des courbes standard et de celle établie sur l'échantillon) nous donne une idée de la variabilité des résultats obtenus pour différentes concentrations de l'échantillon :

$\begin{array}{llllll}\text { Échantillon } \ldots \ldots \ldots \ldots \ldots \ldots \ldots \ldots \ldots \ldots \ldots \ldots \ldots \ldots & 0,2 & 0,4 & 0,6 & 0,8 & \text { I } \\ \text { Luzeme } 2,5 \% \ldots \ldots \ldots \ldots \ldots \ldots\end{array}$

Les chiffres sont en $\%$ du maximum de la courbe standard par cc et sont rapportés à I cc d'hydrolysat dilué. $\sigma=4,7 \quad \bar{\mu}=97 \quad \sigma^{\prime}=\mathbf{I}, 6$

Calcul en tenant compte des dilutions

$$
\frac{97 \times 12,5 \times 100}{60}=2,02 \mathrm{mg} / \text { gramme }
$$

si $\mathrm{P}=0,05$

$$
\frac{2 \times \mathrm{r}, 6 \times \mathrm{I} 2,5 \times 100}{60}=0,07 \quad \text { donc } \quad 2,02: 0,07 .
$$

Quand aucune inhibition ne se manifeste, cette méthode allie à une meilleure spécificité, une précision analogue à la précédente $(3,5 \%$ environ), satisfaite pour plusieurs dosages opérés dans les mêmes conditions. La méthode est cependant limitée au dosage de la méthionine dans les tourteaux, les levures et les fourrages les plus riches en matières azotées.

\section{LACTOBACILLUS FERMENTI 36 (A. T. C. C. 9338)}

L'étude théorique des besoins de ce microorganisme en aminoacides soufrés a été faite par divers auteurs : DUNN (I5), BARTON-WRIGHT (5), Evans (I8), en utilisant des milieux différents. Tous admettent que la L-méthionine est indispensable à Lactobacillus fermenti mais la Dméthionine a la même activité (Dunn (I5) avec le milieu C (Dunn (I4). 
De plus, Evans (18) utilisant le même milieu trouve que l'homocystine avec ou sans choline, ne provoque aucune croissance du microorganisme, et inhibe même celle due à la présence de méthionine. Avec le même milieu employé par ces auteurs, nous avons vérifié que la D-méthionine a la même activité que la forme $\mathrm{L}$, et nous avons fait l'étude de l'activité des dérivés d'oxydation (tableau VI).

\section{TABLEAU VI}

Activité comparée de la L-méthionine, de la DL-méthionine et de la DL-méthioninesulfoxyde pour la croissance de Lactobacillus fermenti 36. Volume total $3 c c, 48$ heures d'incubation.

\begin{tabular}{|c|c|c|c|c|c|}
\hline $\begin{array}{l}\text { L-Méthionine } \\
\mu \mathrm{g}\end{array}$ & $\begin{array}{c}\mathrm{cc} \mathrm{NaOHI} \\
0,03 \mathrm{~N}\end{array}$ & $\begin{array}{c}\text { DL-Méthionine } \\
\text { u.g }\end{array}$ & $\underset{0,03 N}{\operatorname{cc~} \mathrm{NaOH}}$ & $\begin{array}{l}\text { DL-Méthionine } \\
\text { Sulfoxyde } \\
\text { :Lg }\end{array}$ & $\begin{array}{c}\mathrm{cc} \mathrm{NaOII} \\
0,0_{3} \mathrm{~N}\end{array}$ \\
\hline $\begin{array}{r}0 \\
5 \\
10 \\
15 \\
20 \\
25\end{array}$ & $\begin{array}{l}\mathbf{I}, 4 \\
2 \\
2,9 \\
3,75 \\
4,8 \\
5,4\end{array}$ & $\begin{array}{r}0 \\
5 \\
10 \\
15 \\
20 \\
25 \\
30 \\
40 \\
50\end{array}$ & $\begin{array}{c}\mathrm{I}, 4 \\
2,2 \\
3,0 \\
3,45 \\
4,7 \\
5,6 \\
6,7 \\
\text { I0 } \\
\text { I } 2,35\end{array}$ & $\begin{array}{c}0 \\
11,3 \\
22,6 \\
\\
34 \\
45,3 \\
56,6\end{array}$ & $\begin{array}{l}\mathbf{I}, 4 \\
3,45 \\
5,0 \\
6, \mathbf{I} \\
7,5 \\
8,45\end{array}$ \\
\hline
\end{tabular}

La DL-méthioninesulfoxyde présente une activité comparable à celle de la DL-méthionine jusqu'à $30 \mathrm{jg}$ environ, puis elle devient un peu plus faible aux doses plus élevées. Par contre, la DL-méthioninesulfone (tableau VII) n'a aucune activité en la présence ou en l'absence de DL-méthionine.

\section{TABLEAU VII}

Influence de la DL-méthioninesulfone sur la croissance de Lactobacillus fermenti 36 .

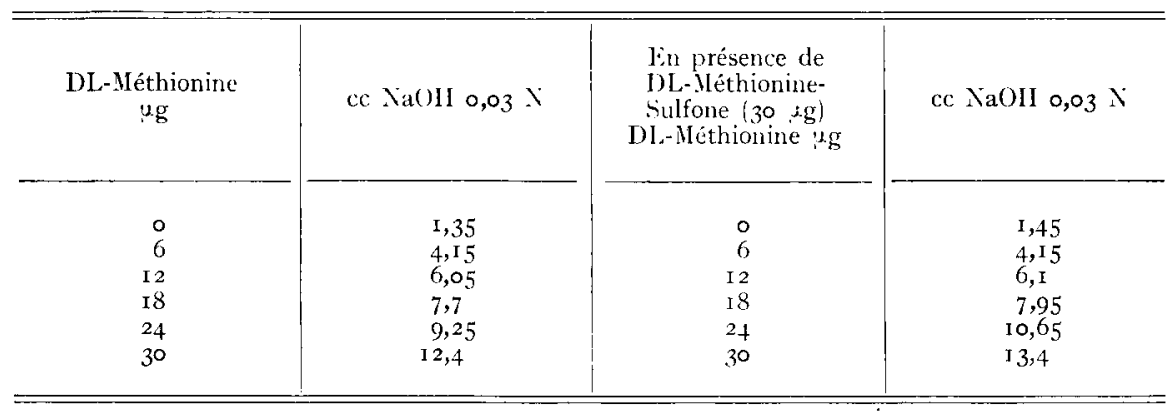

On peut donc conclure que la méthionine est indispensable à la croissance du L. fermenti, mais la spécificité du besoin en cet acide aminé 
est moins étroite que celle de $L$. mésenteroides, car il utilise indistinctement la D-méthionine, la L-méthionine et la DL-méthioninesulfoxyde.

Mode opératoire et précision de la méthode. - La méthode obtenue en utilisant ce microorganisme et le milieu C de DUNN (I4) dont nous avons parlé plus haut, est moins sensible que les précédentes 3 à $30 \mathrm{ug}$ pour une valeur totale de $3 \mathrm{cc}$ (tableau VII). Elle nous a cependant

\section{TABLEAU VIII}

Dosage microbiologique de la $\mathrm{L}-$-méthionine dans les échantillons divers. Comparaison des résultats obtenus par les différents microorganismes.

\begin{tabular}{|c|c|c|c|c|c|c|c|}
\hline \multirow[b]{2}{*}{ Echantillons } & \multirow[b]{2}{*}{$\mathrm{N} \%$} & \multicolumn{2}{|c|}{ L. arabinosus } & \multicolumn{2}{|c|}{ L. mesenteroïdes } & \multicolumn{2}{|c|}{ L. fermenti $3^{6}$} \\
\hline & & $\mathrm{mg} / \mathrm{g}$ & $\mid \begin{array}{c}0 \\
\text { protéines } \\
(16: 0 \mathrm{~N})\end{array}$ & $\operatorname{lng} / \mathrm{g}$ & $\mid \begin{array}{cc}\% \\
\text { protéines } \\
\mathrm{1} 6 \% & \%\end{array}$ & $\mathrm{mg} / \mathrm{g}$ & $\begin{array}{c}\% \\
\text { protéines } \\
(16 \% \mathrm{~N})\end{array}$ \\
\hline (Ovalbumine (O.S. I.) ... & I3 & $3^{1}$ & 3,9 & 34,7 & 4,4 & $3^{2}$ & 3,95 \\
\hline mercial) .............. & 9 & 5 & 0,89 & 5,8 & 1,03 & 6,0 & 1,06 \\
\hline $\begin{array}{c}\text { Luzerne farine commer- } \\
\text { ciale) } \ldots \ldots \ldots \ldots \ldots\end{array}$ & 2,5 & $I, 93$ & $\mathrm{I}, 24$ & 2,02 & 1,30 & $2, \mathrm{I}$ & 1,34 \\
\hline Arachide (traitée au labo- & & & & & 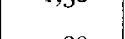 & . & דנילו \\
\hline ratoirel $\ldots \ldots \ldots \ldots$ & 7,75 & 3,50 & 0,73 & 4,3 & 0,88 & 4,3 & 0,88 \\
\hline
\end{tabular}

Remarques: 2 séries d'hydrolysats ont été utilisés l'un pour le dosage de la méthionine à l'aide du Laclobacillus arabinosus et l'autre pour ce même dosagge à l'aide des 2 autres microorganismes.

permis d'obtenir des résultats cohérents pour l'analyse de quelques échantillons (tourteaux et fourrages). I'analyse statistique des résultats a été faite comme il a été signalé plus haut et elle a donné les rés ultats suivants sur un tourteau d'arachide à $9 \mathrm{p}$. cent d'N.

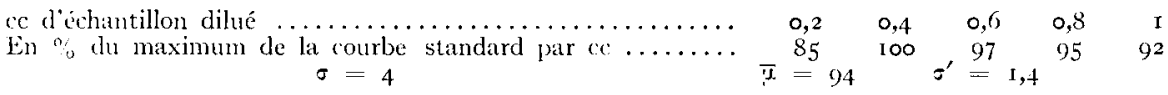
Calcul

$$
\frac{94 \times 16 \times 4}{103}=6,0 \text { g/gramme de produit }
$$

si $\mathrm{P}=0,05$

$$
\frac{2 \times \mathrm{I}, 4 \times 16 \times 4}{103}=0,18 \quad \text { donc } \quad 6,0 \pm 0,18
$$

donc la variabilité des résultats obtenus pour des concentrations croissantes de l'échantillon est du même ordre que pour les autres méthodes \pm 3 p. cent environ. 


\section{Comparaison des résultats}

On peut conclure de cette étude que L. mésenteroides $P$. 60 est le plus spécifique des trois microorganismes, car il n'utilise que faiblement la DL-méthioninesulfoxyde (20 p. cent de l'activité de la DL-méthionine); de plus ses exigences en méthionine semblent indépendantes du milieu et du mode de culture utilisé. Cependant son emploi n'a pu être généralisé, comme nous l'avons déjà indiqué, et nous avons dû employer, plus généralement, le Lactobacillus arabinosus avec le milieu de MarraDEVAN enrichi en vitamines $B$. Ce milieu modifié a donné des résultats cohérents dans tous les échantillons étudiés. Lactobacillus fermenti 36 fournit une méthode moins sensible que les précédentes et de spécificité analogue à celle du Lactobacillus arabinosus; bien que ses exigences soient plus strictes que celles de ce dernier, elle n'a été utilisée qu'à titre de contrôle.

Conditions d'hydrolyse. - Divers auteurs BAILEY (2), BAKAY (3), LugG (24), Fromageot (I9), Singleton (34) entre autres, ont signalé des pertes en méthionine pendant l'hydrolyse acide des échantillons; aussi préconisent-ils 1'emploi de 1'acide formique (Io p. cent à $50 \mathrm{p}$. cent dans l'acide chlorhydrique) comme agent de celle-ci. L'emploi de méthodes microbiologiques de dosage interdit cette méthode, et comme l'acide sulfurique provoque une racémisation importante de la méthionine, nous avons utilisé l'acide chlorhydrique $6 \mathrm{~N}$ qui avait été retenu pour le dosage de la cystine dans les végétaux. Nous avons fait varier le temps d'action à la température de $108^{\circ}$, les hydrolyses étant faites en tube scellé sous vide avec $\mathrm{HCl} 6 \mathrm{~N}$ saturé d'azote pour éviter les risques d'oxydation. Les résultats sont condensés dans le tableau IX et ont

\section{'TABLEAU IX}

Dosage microbiologique de la L-méthionine dans des échantillons divers, hydrolysés pendant des temps variables, en présence d' $\mathrm{HCl} 6 \mathrm{~N}$ en $\%$ des Protéines à $\mathrm{x} 6$ p. cent d'Azote.

\begin{tabular}{|c|c|c|c|}
\hline $\begin{array}{l}\text { Durée de l'hydrolyse } \\
\qquad \grave{a} 108^{11}\end{array}$ & $\begin{array}{c}\text { Lerure de distillerie } \\
5,8 \text { o d'N }\end{array}$ & Arachide $9 \%$ d' & Luzerne $2,5 \%$ d's \\
\hline 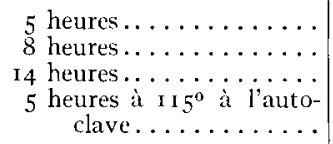 & $\begin{array}{l}1,60 \\
1,56 \\
1,33 \\
1,63\end{array}$ & $\begin{array}{l}0,82 \\
0,85 \\
0,80\end{array}$ & $\begin{array}{l}1,28 \\
I, 30 \\
I, 20\end{array}$ \\
\hline
\end{tabular}

été obtenus par Lactobacillus arabinosus. On peut conclure qu'un temps d'hydrolyse de 5 à 8 heures convient pour les produits analysés et nous 
avons utilisé généralement 7 heures comme nous l'avions fait pour la cystine (ChevilLaRd (I2)).

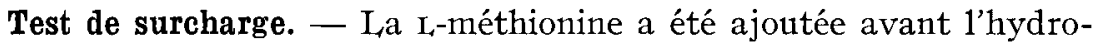
lyse de divers échantillons (ovalbumine, tourteau d'arachide et luzerne) et les dosages de méthionine ont été faits sur chaque échantillon (norma1 et surchargé) et sont condensés dans le tableau X. Les pertes de méthionine libre sont négligeables dans les conditions d'hydrolyse mentionnées plus haut. Ces résultats n'apportent toutefois pas une réponse à la question des pertes qui peuvent se produire dans les combinaisons peptidiques.

TABleau $\mathrm{X}$

Test de surcharge.

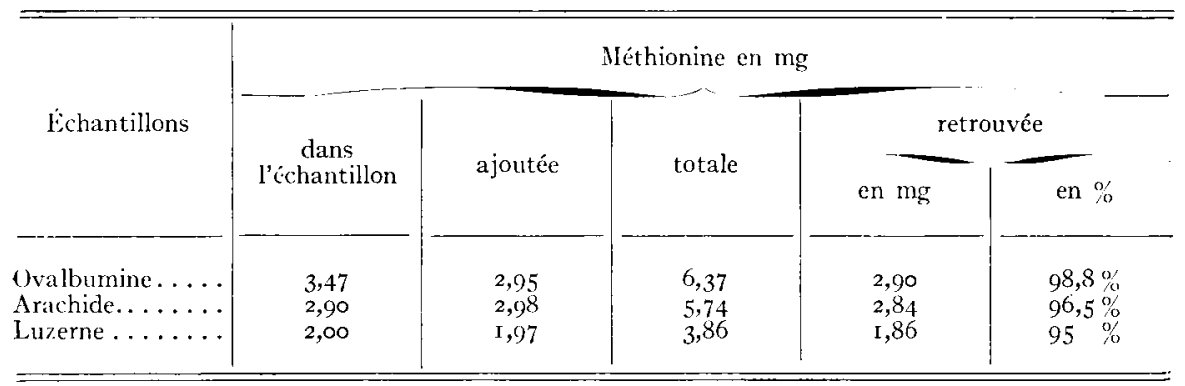

\section{RESULTATS ET CONCLUSIONS}

Les résultats obtenus sur divers échantillons de levures, céréales, tourteau et fourrages satisfont aux critères d'application des méthodes microbiologiques (SNELL, 35 et 36), y compris l'accord entre les résultats obtenus par différents microorganismes pour les produits examinés (tableau VIII).

On peut donc affirmer que la méthionine est effectivement dosée et que son produit d'oxydation (sulfoxyde) ne l'est pas simultanément.

I es résultats obtenus (tableau XI) sur des luzernes de maturités croissantes ne traduisent pas l'existence de différences significatives, ce qui est en accord avec les résultats de LUGG sur le trèfle (25). D'autre part, les graminées et les légumineuses ne semblent pas présenter de différences significatives de concentrations en méthionine.

Par ailleurs, les protéides extraits, représentant 60 à $70 \mathrm{p}$. cent des matières azotées totales, renferment de la méthionine à un taux du même ordre que celles du produit brut si l'on tient compte de la présence d'une grande quantité d'azote non protéique (amide et ammo- 


\section{TABLEAU XI}

Dosage de la L-méthionine dans divers aliments du bétail.

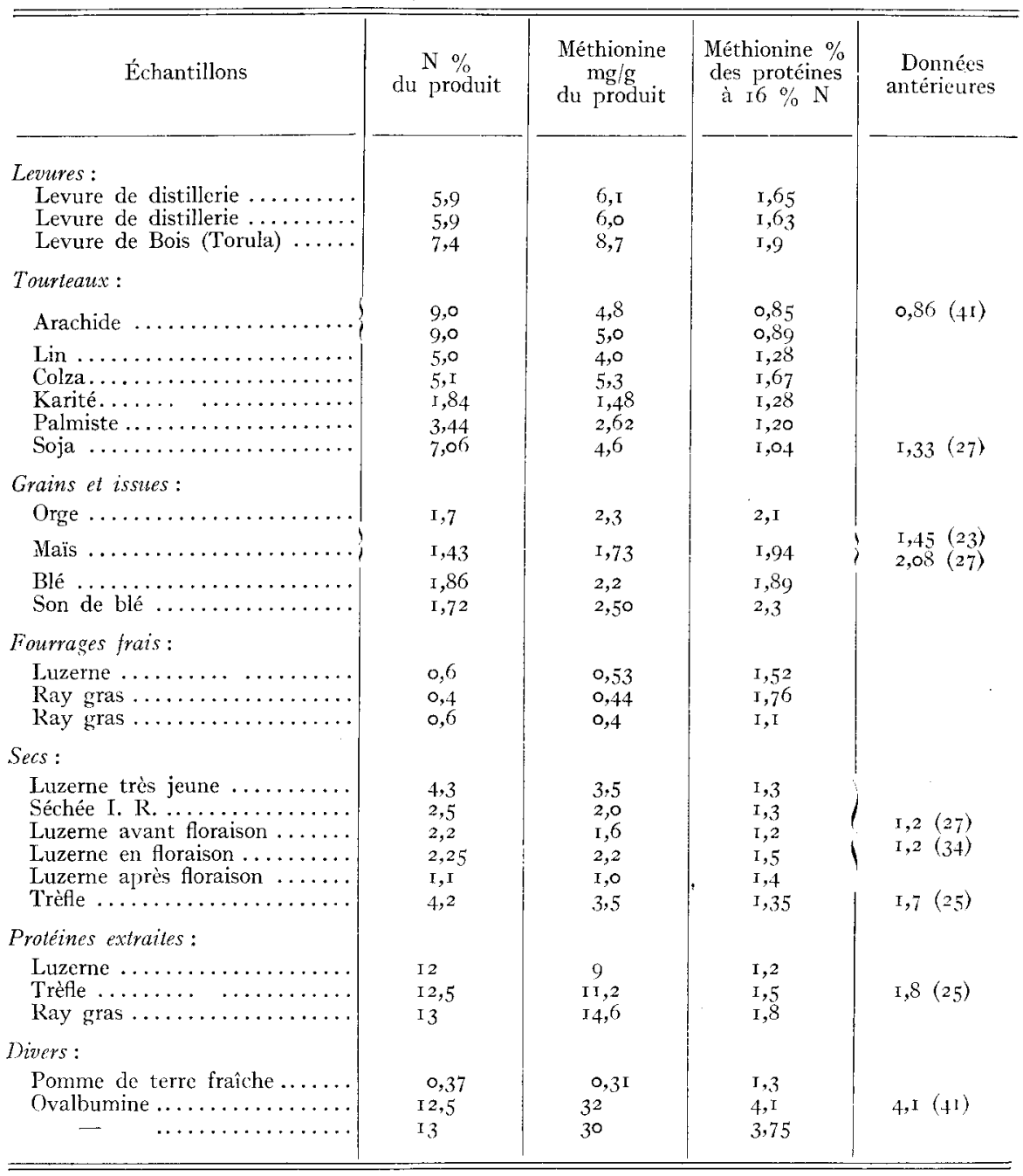

niac) dans les matières azotées résiduelles. Il est donc probable que les pertes en méthionine au cours de l'hydrolyse des produits bruts sont faibles.

Les concentrations en méthionine trouvées dans le matériel étudié, plus faibles que celles obtenues exclusivement dans l'analyse des feuilles, sont en accord avec les résultats de SINGLETON (34) et d'ArMSTRONG (I). 


\section{BIBLIOGRAPHIE}

(I) Armstrong (R. H.). - Amino acids in the protein of herbage. J. Sci. Food. agr. (G. B.), 2, I66-I70, I95I.

(2) Barley (K.). - The sulphur distribution of proteins. Biochem. J., 31 , I307-I 405, I 937 .

(3) Bakay (B.) and Toennies (G.). - Spectrophotometric microdetermination of methionine. J. Biol. Chem., 188, I-I6, I95I.

(4) Barton-Wright (E. C.), EMERy (W. B.) and Robinson (F. A.). - The assay of methionine, lysine, phenylalanine, aspartic acid and proline with Leuconostoc mesenteroides P. 60. Nature, 157, 628-637, I946.

(5) BARTON-WRight (E. C.) and Moran ('T.). - The mictobiological assay of amino acids : cystine, methionine, lysine and threonine. The Analyst, r1, $267-278$, I946.

(6) Brock (R. J.) and Bolling (D.). - The amino acid composition of proteins and foods, I vol. Springfield, I95r, Thomas Ed.

(7) Brickson (W. L.), Henderson (L. M.), SOlHJElL (I.) and Elvehjem (C. A.). - Antagonism of amino acids in the growth of lactic acid bacteria. J. Biol. Chem., 176, 517-528, I948.

(8) Camien (M. N.) and Dunn (M. S.). - The utilization of D-méthionine by Lactobacillus arabinosus I7/5. J. Biol. Chem., 182, II9-I25, I950.

(9) Camien (M. N.) and Dunn (M. S.). - An improved microbiological method for the determination of cystine in human urine with Leuconostoc mesenteroides P. 60. J. Biol. Chem., 183, 56I-568, I950.

(Io) Camien (M. N.) and DunN (M. S.). - Antagonisms in the utilization of D-amino acids by lactic acid bacteria. J. Biol. Chem., 184, 283-288, I950.

(II) Camien (M. N.) and DunN (M. S.). - Antagonisms in the utilization of D-amino acids by lactic acid bacteria. III. Influence of LL-méthionine sulfone and oxidized casein on the utilization of D-methionine. J. Biol. Chem., 18\%, 365-368, I950.

(i2) Chevillard (L.), Fauconneau (G.), Roche (J.). - Dosage microbiologique de la cystine, application aux produits végétaux utilisés dans l'alimentation du bétail. Annales de I. N.R. A., 3, 3I-44, I952.

(I3) Dunn (M. S.), Shankman (S.), Camien (M. N.), Frankei, (W.) and RockIAND (J.). - The nutrition requirements of Leuconostoc mesenteroides P. 60. J. Biol. Chem., 156, 703-713, I944.

(I4) Dunn (M. S.), Camien (M. N.) and Shankman (S.). - Investigations of aminoacids peptides and proteins. XXIV. The aminoacid requirements of Lactobacillus fermenti 36. J. Biol. Chem., 161, 657-667, r945.

(i5) Dunn (M. S.), Camien (M. N.), Shankman (S.) and Block (H.). -- Investigations of amino acids, peptides and proteins. XXVI. The determination of methionine in protein hydrolysates with Lactobacillus fermenti 36. I. Biol. Chem., 163, 577-586, I946.

(i6) Dunn (M. S.), Camien (M. N.), Shankman (S.) and Block (H.). - Urinary excretion of twelve amino acids by normal male and female subjects measured microbiologically. Arch Biochem., 13, 207-216, I947.

(i7) Dunn (M. S.), Shankman (S.), Camien (M. N.) and Block (H.). - The amino acid requirements of twenty three lactic acid bacteria. J. Biol. Chem., 168, I-23, I947.

(I8) Evans (R. J.). - Sulphur amino acid nutrition of some lactic acidproducing bacteria. Arch. Biochem., 16, 357-360, I948.

(I9) Fromageot (C.) et DF Garilhe (M. P.). - La composition du lyzsoyme en acides aminés II. Acides aminés totaux. Biochim. Biophys. Acta, 4, 509-5I7, I950. 
(20) Frey (K. J.). - Variations in the protein and amino acid contents of different oat varieties. Cereal Chem., 29, 77-8I et I42-I47, I952.

(2I) HENDERSON (L. M.), SNer.L (E. E.). - A uniform medium for determination of amino acids with various microorganisms. J. Biol. Chem., 172, I5-29, I948.

(22) Horn (M. S.), Jones (D. B.), Blum (A. E.). - Microbiological determination of methionine in proteins and foods. J. Biol. Chem., 166, 32I-326, I 946.

(23) HorN (M. S.), Jonks (D. B.) and Blum (A. E.). - Methods for microbiological and chemical determinations of essential amino acids in proteins and foods. U. S. Dept. Agric. Misc., Publ. no 696, January I950, pp. I2. Washington DC.

(24) LUGG (J. W. H.). - Preparation of some protein samples from the fresh leaves of plants and the sulphur distributions of the preparations. Biochem. J., 32, 2Ir4-2I28, I938.

(25) LUGG (J. W.) and WeLleR (R. A.). -- Protein in senescent leaves of Trifolium subterraneum. Partial amino acid composition. Bioch. J., 42, 412-4I4, I948.

(26) I yman (C. M.), Moseley (O.), Wood (S.) and Hale (F.). - Note on the use of hydrogen peroxyde-treated peptone in media for the microbiological determination of amino acids. Arch. Biochem., 10, 427-437, I946.

(27) Lyman (C. M.), Moseitey (O.), Buther (B.), Wood (S.) and Hale (F.). The microbiological determination of amino-acids. III Methionine. $J$. Biol. Chem., 166, I6I-I7I, I947.

(28) Mahadevan (V.). - Contribution à l'étude de la régénération de la fourrure et de la composition des poils du rat blanc. Influence des aminoacides et des iodocaséines. Déductions au problème de la laine. Thèse de Doctorat es Sciences, 23 novembre I950, Paris.

(29) Mondolfa (U.) et Camboni (V.). - Recherche sulla ditributione di alcuni amino acidi. La determinazione degli amino acidi con $\mathrm{i}$ methodi microbiologici. Boll. inst. Ser., Milan, 28, 333-352, I949.

(30) Riesen (W. H.), Schweiger'T (B. S.), EilvehJ EM (C. A.) - Microbiological determination of methionine in proteins and foodstuffs. $J$. Biol. Chem., 165, 347-358, r946.

(3I) Riesen (W. H.), Spengler (H. H.), Robblee (A. R.), Hankes (L. V.) and ELVEHJEM (C. A.). - Cystine and related compounds in the nutrition of lactic acid bacteria. J. Biol. Chem., 1\%1, 73I-748, I947.

(32) Sauberitich (H. E.) and Baumann (C. A.). - The effect of dietary protein upon amino acid excretion by rats and mice. J. Biol. Chem., 166, $4 \mathrm{I} 7-428$, I 946 .

(33) Shankman (S.), Dunn (M. S.), and Rubin (L. B.). - Assay of 8-aminoacids in amino acid test mixture using Lactobacillus arabinosus $\mathbf{I} 7 / 5$. J. Biol. Chem., 150, 477-48I, I943.

(34) Singleton (K. L.), MeRTZ (E. T.) and Davis (R. L.). - The hydrolysis and amino acid assay of alfafa and the methionine range in Ioo selections. Agronomy Journal (N. Y.), 44, 346-348, I952.

(35) SNeir (E. E.). - The microbiological Assay of amino-acids Adv. in Protein Chemistry, II, 85-II 8, I945.

(36) SNELL (E. E.) and Schweigber'T (B. S.). - Microbiological methods for the estimation of amino acids. Nutr. Abstr. and Rev., 16, 497-509, I947.

(37) Spies (J. R.) and Chambers (D. C.). - Utilization of optical isomers of methionine and formylmethionine by some Lactobacilli. J. Biol. Chem. 183, 709-7I2, I950.

(38) Steele (B. F.), Sauberlich (H. E.), Reynolds (M. S.) and Baumann (C. A.). - Media for Leuconostoc mesenteroides P. 60 and Leuconostoc citrovorum 8081. J. Biol. Chem., 1\%\%, 533-544, I949. 
(39) Stokfs (S. L.), Gunnes (M.), Dwyer (I. M.) and Casweit (M. S.). Determination of Io essential amino acids phenylalanine with $\mathrm{L}$. delbruckii and the other nine with S. Foecalis. J. Biol. Chem., 160, 35-49, I945.

(40) Woodson (H. W.), Hier (S. W.), Solomon (J. D.) and Bergeim (O.). Urinary excretion of amino acids by human subjects on normal diets J. Biol. Chem., 1\%2, 613-6I8, I948.

(4I) Cooperative determination of the amino acid content and of the nutritive value of six selected protein food sources, I946-I950, Rutgers University, New Brunswick, New Jersey, I95I. 